\title{
Direct esterification of cinnamic acids with phenols and imidoalcohols: a simple, heteropolyacid-catalyzed procedure
}

\author{
Diego M. Ruiz, ${ }^{\text {a,b }}$ Gustavo P. Romanelli, ${ }^{\text {a,b,* }}$ Daniel O. Bennardi, ${ }^{\text {a,b }}$ Graciela T. Baronetti, \\ Horacio J. Thomas, ${ }^{b}$ and Juan C. Autino ${ }^{\mathrm{a}, *}$ \\ ${ }^{a}$ Cátedra de Química Orgánica, Facultad de Ciencias Agrarias y Forestales, Universidad \\ Nacional de La Plata. Calles 60 y 119, B1904AAN La Plata, República Argentina \\ ${ }^{b}$ Centro de Investigación y Desarrollo en Ciencias Aplicadas "Dr. Jorge J. Ronco" \\ (CINDECA), Departamento de Química, Facultad de Ciencias Exactas, Universidad Nacional \\ de La Plata-CONICET. Calle $47 N^{\circ}$ 257, B1900AJK La Plata, República Argentina \\ ${ }^{c}$ Facultad de Ingeniería, Universidad de Buenos Aires. Buenos Aires, República Argentina \\ E-mail:gpr@agro.unlp.edu.ar
}

\begin{abstract}
A convenient procedure for the direct esterification of cinnamic acids with phenols or 2- $(N-$ phthalimido)ethanol is described. The method is simple and clean, environmentally friendly and high-yielding for both electron-releasing and electron-withdrawing substituted phenols. The heteropolyacid $\mathrm{H}_{6} \mathrm{P}_{2} \mathrm{~W}_{18} \mathrm{O}_{62} \cdot 24 \mathrm{H}_{2} \mathrm{O}$ was employed as catalyst, both bulk and supported on silica. No stoichiometric activation of the carboxyl group or condensing reagents is necessary. The effects of temperature, reaction time, amount of the catalyst used and mole ratio of the reactants on the ester yield were checked. Suitable conditions for the reaction include a 1:1 molar ratio of reactants and a $1 \mathrm{mmol} \%$ mass ratio of catalyst to reactant. Eighteen aryl and phthalimidoethyl cinnamates were obtained, yields ranged in $84-95 \%$ for most of the esters. The catalyst shown to be reusable for at least three times without occurring an appreciable loss of its activity.
\end{abstract}

Keywords: Acid catalysis, cinnamates, direct esterification, heteropolyacid, Wells-Dawson acid

\section{Introduction}

Cinnamates are both antioxidant and/or flavoring agents in diverse plant species. A bioactive imidoalkyl cinnamate, 2-(N-phthalimido)ethyl cinnamate, is a potent inhibitor of the 17ß-hydroxysteroid dehydrogenase enzymes involved in diseases such as prostatic and breast cancer, Alzheimer disease and benign prostatic hyperplasia. ${ }^{1}$ Aryl cinnamates have been used as intermediates for diverse heterocyclic compounds, for example benzofuranones, ${ }^{2}$ styryl chromones and pyrazoles. ${ }^{3}$ Some substituted aryl cinnamates are antifungal ${ }^{1}$ and inhibitory of plant growth. ${ }^{4}$ 
Only a few procedures have been used to prepare aryl cinnamates, including the classic method via cinnamoyl chloride, ${ }^{5}$ or using $\mathrm{DCC}^{6}$ or $\mathrm{BOP}^{1}$ as coupling agents to perform the direct reaction between cinnamic acid and a phenol; or from a phosphorane and a benzaldehyde ${ }^{7}$. However, these methods have drawbacks, such as the formation of the carcinogenic by-product HMPA (from BOP) ${ }^{8}$ or, from the point of view of green chemistry, a low atom economy (by using $\mathrm{DCC}$ or $\mathrm{SOCl}_{2}$; besides, in the latter case a heating at $350^{\circ}$ is prescribed)..$^{5}$ Other cinnamates have been obtained using friendly conditions from cinnamaldehydes using heterogeneous catalyst and $\mathrm{DDQ}^{9}$, and direct esterification using water-tolerant $\mathrm{ZrOCl}_{2} .8 \mathrm{H}_{2} \mathrm{O}$ and $\mathrm{HfOCl}_{2} .8 \mathrm{H}_{2} \mathrm{O}$ as catalysts ${ }^{10}$.

This work was carried out in the course of our research project for the environmentally friendly synthesis of selective pesticides. Up to now, and to the best of our knowledge, no report has been made about a simple and clean methodology for carrying out the direct esterification between phenols and $\alpha, \beta$ unsaturated acids. A few procedures have been reported for performing the acetylation of alcohols with the aid of some heteropolyacid catalysts: starting from acetic anhydride and primary or secondary alcohols ${ }^{11}$ or from acetic acid and primary, secondary, tertiary or benzylic alcohols. ${ }^{12}$

The use of solid heteropolyacids (HPAs) is convenient because of their redox and superacidic properties, and because the reaction mixture involves a heterogeneous catalysis. These characteristics turn HPAs into useful and versatile catalysts for a number of transformations; ${ }^{13,14}$ both an easy and clean workup of the product and the recycling of the catalyst can be achieved. The molecular structure of the WellsDawson (WD) heteropolyacid catalyst $\left(\mathrm{H}_{6} \mathrm{P}_{2} \mathrm{~W}_{18} \mathrm{O}_{62} \cdot 24 \mathrm{H}_{2} \mathrm{O}\right)^{15,16}$ shows two identical "half units" $\mathrm{PW}_{9}$ linked through the oxygen atoms, and consists of a close-packed framework of W(VI)-oxygen octahedra $\mathrm{WO}_{6}$ surrounding a central $\mathrm{P}(\mathrm{V})$ atom. Wells-Dawson HPA has been reported as a useful catalyst for the sustainable synthesis of various organic compounds, for example methyl tert-butyl ether, ${ }^{15}$ 1,1,3-trimethyl3-phenylindan, ${ }^{17} 4$-substituted 1,3-dioxanes, ${ }^{18}$ and coumarins. ${ }^{19}$ Besides, WD acid as been used to perform reactions, reviews including this matter have been published recently. ${ }^{20,21}$ Over the last years WD-catalyzed reactions have been reported, mention can be made of the epoxidation of alkenes and alkenols, ${ }^{22}$ preparation of 1,3-dioxolane derivatives by the reaction of epoxides with carbonyl compounds; ${ }^{23}$ protection of alcohols and phenols as tetrahydropyranyl ethers and their deprotection, cleavage of MOM-ethers of phenols, preparation of acylals ${ }^{19}$ and their cleavage, ${ }^{24}$ preparation of benzhydryl ethers of alcohols, ${ }^{25}$ and acetylation of alcohols. $^{12}$

\section{Results and Discussion}

In connection with our research project on the eco-friendly synthesis of organics related to selective pesticides, we wish to report the results obtained for the direct esterification between cinnamic acids (1) and phenols (2) or 2-( $N$-phthalimido)ethanol (3) (see Scheme 1). Wells-Dawson HPA was employed as an ecofriendly, reusable catalyst in both bulk and silica-supported forms; in both cases heterogeneous catalysis takes place. Structures and the obtained results are shown in Table 1. To optimize the reaction, cinnamic acid and phenol were used as substrates; temperature, mole ratio of substrates to WD acid, concentration of the solution, and reaction time were varied. All products were identified by comparison of analytical, 
physical and spectral data with those reported, or with authentic samples prepared following the conventional method.
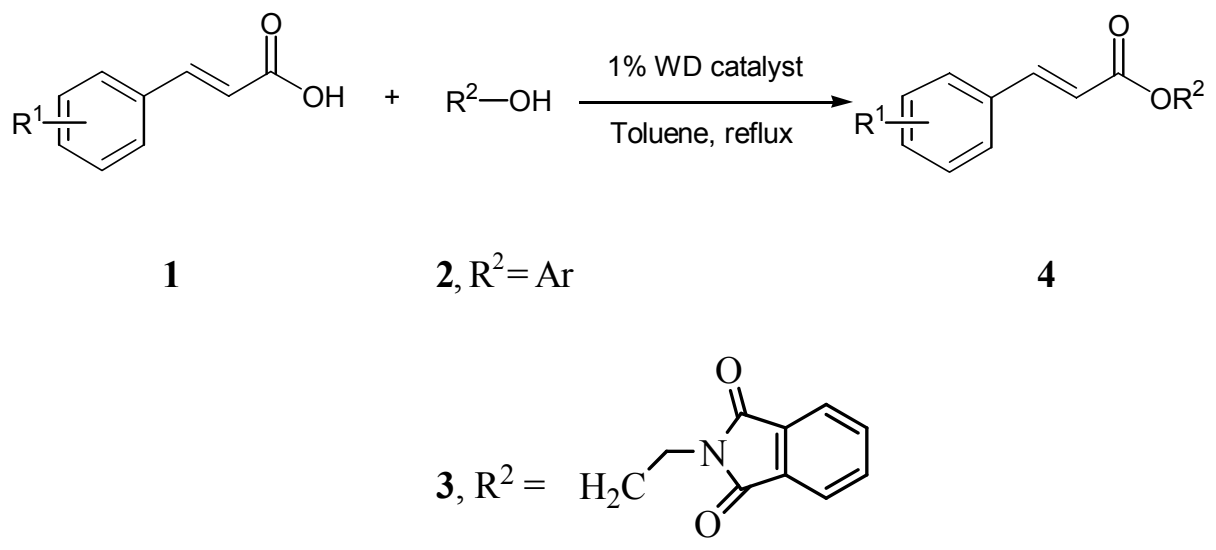

\section{Scheme 1}

The WD acid was prepared as described elsewhere ${ }^{13}$, from an aqueous solution of $\alpha / \beta \mathrm{K}_{6} \mathrm{P}_{2} \mathrm{~W}_{18} \mathrm{O}_{62} \cdot 10$ $\mathrm{H}_{2} \mathrm{O}$ salt, which was treated with ether and concentrated (37\%) HCl solution. Silica-supported WD acid was obtained by wet impregnation of Grace Davison silica (Grade 59, specific area $=250 \mathrm{~m}^{2} / \mathrm{g}$ ) with an aqueous solution of WD acid; a catalyst containing 40\% (by weight) of WD acid was prepared. After impregnation, catalyst samples were dried at room temperature in a vacuum desiccator for $8 \mathrm{~h}$.

The optimization experiments were performed until one of the reactants was consumed, or until no changes in the composition of the reaction mixture were observed. The reaction proceeds smoothly in refluxing toluene using WD acid supported on silica; after 1\% (mmol) of supported WD acid was added to the solution of reactants, the highest yield of phenyl cinnamate (see Table 1, entry 1) was reached after 120 min of reaction. The use of $10 \%$ (mmol) of supported WD acid did not improve the result; longer reaction times caused a lowering of the yield.

When the optimized conditions were applied to the substituted phenolic substrates, high yields of cinnamates were attained; the electrical nature of the substituents seems to have no great effects on the reaction yield (see Table 1, entries 2, 4, 5, 10). Typical examples were reacted in the presence of each of the catalysts. When bulk WD acid is used, yield and conversion values are very similar to that obtained with supported WD acid, but double time is required for attaining comparable yields. Thymol (see Table 1, entry ) gives a good yield in $2 \mathrm{~h}$ reaction, similarly to p-nitrophenol (Table 1, entry 5). Paracetamol did not react with cinnamic acid nor with 3,4,5- trimethoxycinnamic acid, after $5 \mathrm{~h}$ at $110^{\circ} \mathrm{C}$ (not cited in Table 1). However, phenyl 3,4,5-trimethoxycinnamate was obtained in $22 \%$ yield, $95 \%$ conversion, in $5 \mathrm{~h}$ reaction at $110^{\circ} \mathrm{C}$; a longer reaction time caused a yield decrease. 2-Phthalimidoethanol reacts with cinnamic acid giving ester (see Table 1, entry 13) slower than the previously discussed examples; this could be due to the protonation of the imide at an $\mathrm{O}$ atom. Substitution at the cinnamic acid gives comparable yields to that of the unsubstituted acid. Catalyst recycling was checked in two consecutive batches after their first use (see Table 1, entries 1 and 11) showing that the catalyst maintains an almost constant activity. 
Table 1. Catalytic preparation of aryl and phthalimidoethyl cinnamates ${ }^{\mathrm{a}}$

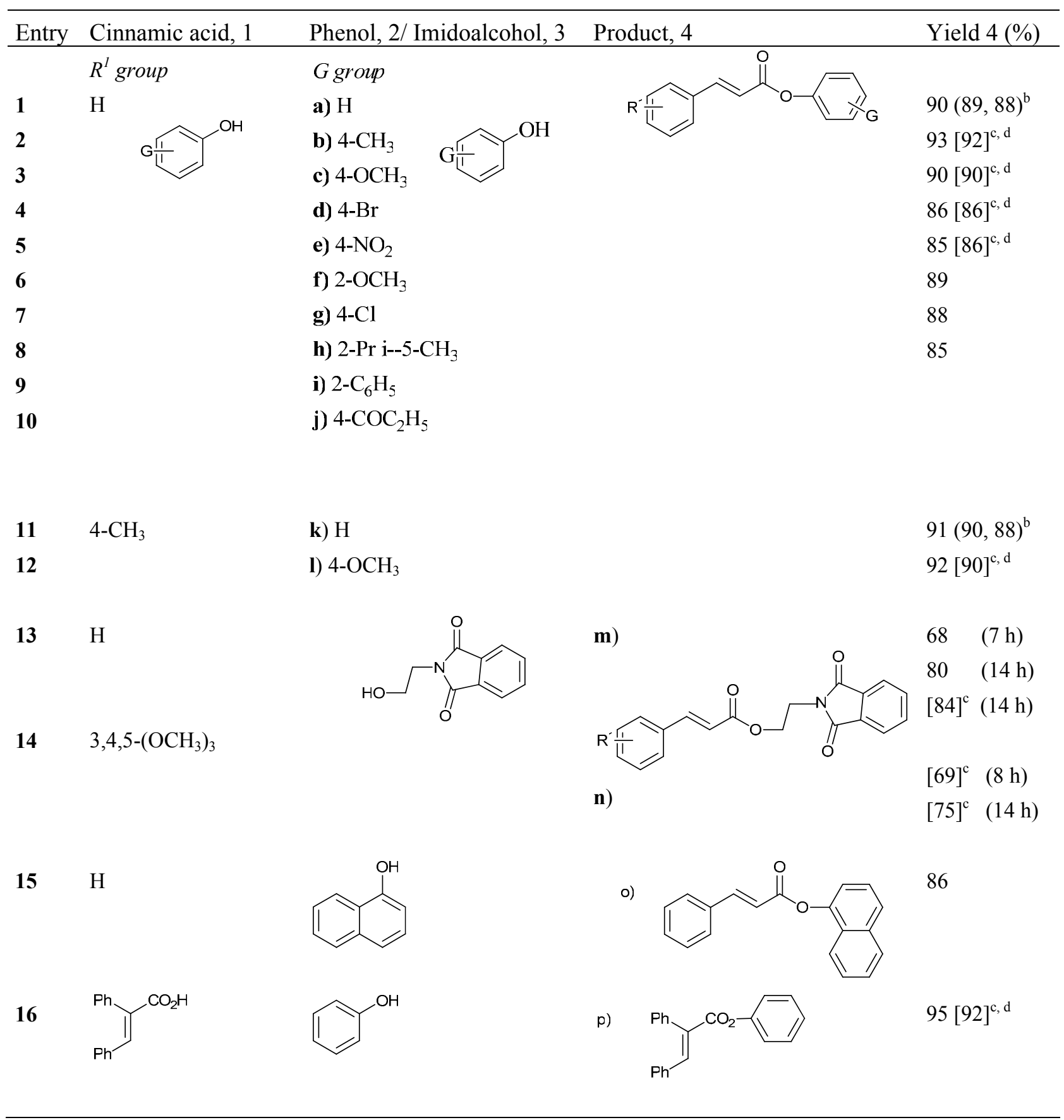

${ }^{a}$ Reactions were carried out in refluxing toluene, using $1 \%$ (mmol) of WD acid. Reaction time was $2 \mathrm{~h}$ and WD acid is supported (40\%) on silica, except when otherwise stated. ${ }^{\mathrm{b}}$ Yields obtained in the first and second reuse of the recovered catalyst. ${ }^{\mathrm{c}}$ Yield obtained when bulk WD acid was used. ${ }^{\mathrm{d}}$ Reaction time was $4 \mathrm{~h}$. 


\section{Experimental Section}

General Procedures. All melting points were determined with a Büchi Melting Point equipment and are uncorrected. Elemental microanalyses were performed in F \& $\mathrm{M}$ instrument. ${ }^{1} \mathrm{H}$ NMR spectra $(200,250$ or $400 \mathrm{MHz})$ and ${ }^{13} \mathrm{C}-\mathrm{NMR}$ spectra $(50,62,5$ or $100 \mathrm{MHz})$ were recorded on Varian Mercury-200, Bruker Gemini 2000 or Bruker GX-400 spectrometers, respectively. Chemical shifts $(\delta)$ are given from TMS $(0 \mathrm{ppm})$ as internal standard for ${ }^{1} \mathrm{H}-\mathrm{NMR}$, and ${ }^{13} \mathrm{CDCl}_{3}$ (77.0 ppm) for ${ }^{13} \mathrm{C}-\mathrm{NMR}$.

\section{General procedure for preparing aryl and imidoethyl cinnamates}

A solution of a cinnamic acid $\mathbf{1}(1.1 \mathrm{mmol})$ and a phenol $\mathbf{2}$ or 2-phthalimidoethanol $3(1 \mathrm{mmol})$ in toluene ( $2 \mathrm{ml}$, or $3 \mathrm{ml}$ when supported catalyst was used) and the bulk or silica-supported WD acid $\left(10^{-2} \mathrm{mmol}\right)$ were refluxed with stirring for the indicated time. In both cases the catalyst was removed by filtration and washed twice with toluene $(1 \mathrm{~mL}$ each). The organic solution was washed with cold $1 \mathrm{M} \mathrm{NaOH}(2 \times 2 \mathrm{~mL})$ and $\mathrm{H}_{2} \mathrm{O}(2 \times 2 \mathrm{~mL})$ and then dried over anh. $\mathrm{Na}_{2} \mathrm{SO}_{4}$. Evaporation of the solvent under reduced pressure and recrystallization from hexanes, or silica flash column chromatography gave the pure cinnamate 4 .

\section{Compound characterization}

Phenyl cinnamate (4a). ${ }^{1} \mathrm{H}$ NMR (400 MHz, $\left.\mathrm{CDCl}_{3}\right)$ : $\delta 6.64(1 \mathrm{H}, \mathrm{d}, J=16 \mathrm{~Hz}), 7.18(2 \mathrm{H}, \mathrm{br}$ d, $J=7.5 \mathrm{~Hz}), 7.25(1 \mathrm{H}$, br t, $J=7.3 \mathrm{~Hz}), 7.38-7.46(5 \mathrm{H}, \mathrm{m}), 7.56-7.62(2 \mathrm{H}, \mathrm{m}), 7.88(1 \mathrm{H}, \mathrm{d}, J=$ $16 \mathrm{~Hz}) ;{ }^{13} \mathrm{C}$ NMR (100 MHz, $\left.\mathrm{CDCl}_{3}\right): 117.4,121.6,125.8,128.3,129.0,129.4,130.7,134.2$, 146.6, 150.8, 165.4.

4-Methylphenyl cinnamate (4b). ${ }^{26} \mathrm{H}$ NMR $\left(400 \mathrm{MHz}, \mathrm{CDCl}_{3}\right): 2.36(3 \mathrm{H}, \mathrm{s}), 6.62(1 \mathrm{H}, \mathrm{d}, J=$ $16 \mathrm{~Hz}), 7.04-7.06(2 \mathrm{H}, \mathrm{m}), 7.19-7.21(2 \mathrm{H}, \mathrm{d}, J=8.1 \mathrm{~Hz}), 7.41-7.42(3 \mathrm{H}, \mathrm{m}), 7.57-7.59(2 \mathrm{H}, \mathrm{m})$, $7.86(1 \mathrm{H}, \mathrm{d}, J=16 \mathrm{~Hz}) ;{ }^{13} \mathrm{C} \mathrm{NMR}\left(100 \mathrm{MHz}, \mathrm{CDCl}_{3}\right): 20.9,117.4,121.3,128.3,129.0,130.0$, 130.6, 134.2, 135.4, 146.4, 148.6, 165.6.

4-Methoxyphenyl cinnamate (4c). ${ }^{27}{ }^{1} \mathrm{H}$ NMR (400 MHz, $\left.\mathrm{CDCl}_{3}\right): 3.74(3 \mathrm{H}, \mathrm{s}), 6.55(1 \mathrm{H}, \mathrm{d}, J=$ $16 \mathrm{~Hz}), 6.81-6.87(2 \mathrm{H}, \mathrm{m}), 6.99-7.05(2 \mathrm{H}, \mathrm{m}), 7.32-7.38(3 \mathrm{H}, \mathrm{m}), 7.48-7.54(2 \mathrm{H}, \mathrm{m}), 7.78(1 \mathrm{H}$, d, $J=16 \mathrm{~Hz}) ;{ }^{13} \mathrm{C} \mathrm{NMR}\left(100 \mathrm{MHz}, \mathrm{CDCl}_{3}\right): 55.6,114.5,117.4,122.4,128.3,129.0,130.6$, $134.2,144.3,146.4,157.3,165.8$.

4-Bromophenyl cinnamate (4d). ${ }^{28}{ }^{1} \mathrm{H}$ NMR $\left(400 \mathrm{MHz}, \mathrm{CDCl}_{3}\right): 6.60(1 \mathrm{H}, \mathrm{d}, J=16 \mathrm{~Hz}), 7.02-$ $7.10(2 \mathrm{H}, \mathrm{d}, J=8.9 \mathrm{~Hz}), 7.25-7.38(3 \mathrm{H}, \mathrm{m}), 7.40-7.45(2 \mathrm{H}, \mathrm{d}, J=8.8 \mathrm{~Hz}), 7.57-7.62(2 \mathrm{H}, \mathrm{m})$, $7.86(1 \mathrm{H}, \mathrm{d}, J=16 \mathrm{~Hz}) ;{ }^{13} \mathrm{C}$ NMR $\left(100 \mathrm{MHz}, \mathrm{CDCl}_{3}\right): 117.1,119.1,123.7,128.6,129.3,131.1$, 132.7, 134.3, 147.3, 150.1, 165.3.

4-Nitrophenyl cinnamate (4e). ${ }^{29}{ }^{1} \mathrm{H}$ NMR $\left(400 \mathrm{MHz}, \mathrm{CDCl}_{3}\right): 6.63(1 \mathrm{H}, \mathrm{d}, J=16 \mathrm{~Hz}), 7.37$ $(2 \mathrm{H}, \mathrm{d}, J=9 \mathrm{~Hz}), 7.40-7.50(3 \mathrm{H}, \mathrm{m}), 7.55-7.65(2 \mathrm{H}, \mathrm{m}), 7.92(1 \mathrm{H}, \mathrm{d}, J=16 \mathrm{~Hz}), 8.30(2 \mathrm{H}, \mathrm{d}, J$ $=8 \mathrm{~Hz}) ;{ }^{13} \mathrm{C} \mathrm{NMR}\left(100 \mathrm{MHz}, \mathrm{CDCl}_{3}\right): 116.2,122.5,125.2,128.5,129.1,129.5,133.8,148.0$, 155.6, 164.3. 
2-Methoxyphenyl cinnamate (4f). ${ }^{1} \mathrm{H}$ NMR $\left(400 \mathrm{MHz}, \mathrm{CDCl}_{3}\right): 3.88(3 \mathrm{H}, \mathrm{s}), 6.63(1 \mathrm{H}, \mathrm{d}, J=$ $16 \mathrm{~Hz}), 6.89-7.01(2 \mathrm{H}, \mathrm{m}), 7.03-7.09(1 \mathrm{H}, \mathrm{m}), 7.23-7.31(1 \mathrm{H}, \mathrm{m}), 7.39-7.46(3 \mathrm{H}, \mathrm{m}), 7.55-7.62$ $(2 \mathrm{H}, \mathrm{m}), 7.86(1 \mathrm{H}, \mathrm{d}, J=16 \mathrm{~Hz}) ;{ }^{13} \mathrm{C} \mathrm{NMR}\left(100 \mathrm{MHz}, \mathrm{CDCl}_{3}\right): 55.9,112.5,117.1,120.8$, $122.9,126.9,128.3,128.9,130.6,134.3,139.9,146.5,151.3,164.9$.

4-Chlorophenyl cinnamate (4g). ${ }^{28}{ }^{1} \mathrm{H} \mathrm{NMR}\left(400 \mathrm{MHz}, \mathrm{CDCl}_{3}\right): 6.61(1 \mathrm{H}, \mathrm{d}, J=16 \mathrm{~Hz}), 7.08$ $7.12(2 \mathrm{H}, \mathrm{m}), 7.35-7.36(2 \mathrm{H}, \mathrm{m}), 7.41-7.43(3 \mathrm{H}, \mathrm{m}), 7.57-7.59(2 \mathrm{H}, \mathrm{m}), 7.85(1 \mathrm{H}, \mathrm{d}, J=16 \mathrm{~Hz})$; ${ }^{13} \mathrm{C}$ NMR (100 MHz, $\left.\mathrm{CDCl}_{3}\right)$ : 116.9, 123.0, 128.3, 129.0, 129.5, 130.8, 131.1, 134.1, 147.0, 149.3, 165.1 .

2-Isopropyl-5-methylphenyl cinnamate (4h). ${ }^{30} \mathrm{H}$ NMR (400 MHz, $\left.\mathrm{CDCl}_{3}\right): 1.20$ (3H, s), 1.22 $(3 \mathrm{H}, \mathrm{s}), 2.34(3 \mathrm{H}, \mathrm{s}), 3.04(1 \mathrm{H}, \mathrm{m}), 6.67(1 \mathrm{H}, \mathrm{d}, J=16 \mathrm{~Hz}), 6.89(1 \mathrm{H}, \mathrm{br}), 7.04(1 \mathrm{H}, \mathrm{d}, J=8$ $\mathrm{Hz}), 7.22(1 \mathrm{H}, \mathrm{d}, J=8 \mathrm{~Hz}), 7.39-7.48(3 \mathrm{H}, \mathrm{m}), 7.57-7.63(2 \mathrm{H}, \mathrm{m}), 7.88(1 \mathrm{H}, \mathrm{d}, J=16 \mathrm{~Hz}) ;{ }^{13} \mathrm{C}$ NMR (100 MHz, $\left.\mathrm{CDCl}_{3}\right): 116.2,122.5,125.2,128.5,129.1,129.5,133.8,148.0,155.6,164.3$

2-Biphenylyl cinnamate (4i). ${ }^{\text {lit. }}{ }^{11} \mathrm{H} \mathrm{NMR}\left(400 \mathrm{MHz}, \mathrm{CDCl}_{3}\right): 6.47(1 \mathrm{H}, \mathrm{d}, J=16 \mathrm{~Hz}), 7.22$ $7.26(1 \mathrm{H}, \mathrm{dd}, J=8,1.3 \mathrm{~Hz}), 7.28-7.41(8 \mathrm{H}, \mathrm{m}), 7.43-7.47(3 \mathrm{H}, \mathrm{m}), 7.48-7.53(2 \mathrm{H}, \mathrm{m}), 7.70(1 \mathrm{H}$, $\mathrm{d}, J=16 \mathrm{~Hz}) ;{ }^{13} \mathrm{C} \mathrm{NMR}\left(100 \mathrm{MHz}, \mathrm{CDCl}_{3}\right): 117.1,123.0,126.3,127.4,128.3,128.5,128.9$, 130.6, 131.0, 134.2, 134.9, 137.6, 146.5, 147.9, 165.3.

4-Propionylphenyl cinnamate (4j). ${ }^{1} \mathrm{H} \mathrm{NMR}\left(400 \mathrm{MHz}, \mathrm{CDCl}_{3}\right): 1.24(3 \mathrm{H}, \mathrm{t}, J=7 \mathrm{~Hz}), 3.02$ $(2 \mathrm{H}, \mathrm{qt}, J=7 \mathrm{~Hz}), 6.63(1 \mathrm{H}, \mathrm{d}, J=16 \mathrm{~Hz}), 7.27-7.32(2 \mathrm{H}, \mathrm{m}), 7.39-7.48(3 \mathrm{H}, \mathrm{m}), 7.56-7.67(2 \mathrm{H}$, m), $7.99(1 \mathrm{H}, \mathrm{d}, J=16 \mathrm{~Hz}), 8.01-8.08(2 \mathrm{H}, \mathrm{m}) .{ }^{13} \mathrm{C} \mathrm{NMR}\left(100 \mathrm{MHz}, \mathrm{CDCl}_{3}\right): 8.3,31.8,116.8$, 121.8, 128.4, 129.1, 129.6, 130.9, 134.0, 134.5, 147.3, 154.4, 164.8, 199.6. Found: C, 77.10; H, 5.73. $\mathrm{C}_{18} \mathrm{H}_{16} \mathrm{O}_{3}$ requires: $\mathrm{C}, 77.12 ; \mathrm{H}, 5.75$.

Phenyl 4-methylcinnamate (4k). ${ }^{1} \mathrm{H}$ NMR (400 $\left.\mathrm{MHz}, \mathrm{CDCl}_{3}\right): 1.24(3 \mathrm{H}, \mathrm{t}, J=7.3 \mathrm{~Hz}), 3.01$ $(2 \mathrm{H}, \mathrm{qt}, J=7.3 \mathrm{~Hz}), 6.64(1 \mathrm{H}, \mathrm{d}, J=16 \mathrm{~Hz}), 7.26-7.29(2 \mathrm{H}, \mathrm{m}), 7.45-7.43(3 \mathrm{H}, \mathrm{m}), 7.59-7.61$ $(2 \mathrm{H}, \mathrm{m}), 7.90(1 \mathrm{H}, \mathrm{d}, J=16 \mathrm{~Hz}), 8.04(2 \mathrm{H}, \mathrm{d}, J=8.7 \mathrm{~Hz}) .{ }^{13} \mathrm{C} \mathrm{NMR}\left(100 \mathrm{MHz}, \mathrm{CDCl}_{3}\right): 116.2$, 122.5 , 125.2, 128.5, 129.1, 129.5, 133.8, 148.0, 155.6, 164.3. Found: C, 80.63; H, 5.91. $\mathrm{C}_{16} \mathrm{H}_{14} \mathrm{O}_{2}$ requires: $\mathrm{C}, 80.65 ; \mathrm{H}, 5.92$.

4-Methoxyphenyl 4-methylcinnamate (4I). ${ }^{1} \mathrm{H}$ NMR (400 $\left.\mathrm{MHz}, \mathrm{CDCl}_{3}\right): 1.24(3 \mathrm{H}, \mathrm{t}, J=7.3$ $\mathrm{Hz}), 3.01(2 \mathrm{H}, \mathrm{qt}, J=7.3 \mathrm{~Hz}), 6.64(1 \mathrm{H}, \mathrm{d}, J=16 \mathrm{~Hz}), 7.26-7.29(2 \mathrm{H}, \mathrm{m}), 7.45-7.43(3 \mathrm{H}, \mathrm{m})$, 7.59-7.61 (2H, m), $7.90(1 \mathrm{H}, \mathrm{d}, J=16 \mathrm{~Hz}), 8.04(2 \mathrm{H}, \mathrm{d}, J=8.7 \mathrm{~Hz}) .{ }^{13} \mathrm{C} \mathrm{NMR}(100 \mathrm{MHz}$, $\left.\mathrm{CDCl}_{3}\right): 116.2,122.5,125.2,128.5,129.1,129.5,133.8,148.0,155.6,164.3$. Found: C, 76.09; $\mathrm{H}, 5.99 . \mathrm{C}_{17} \mathrm{H}_{16} \mathrm{O}_{3}$ requires: $\mathrm{C}, 76.10 ; \mathrm{H}, 6.01$.

2-Phthalimidoethyl cinnamate (4m). ${ }^{1} \mathrm{H}$ NMR (400 MHz, $\left.\mathrm{CDCl}_{3}\right): 1.24(3 \mathrm{H}, \mathrm{t}, J=7.3 \mathrm{~Hz}$ ), $3.01(2 \mathrm{H}, \mathrm{qt}, J=7.3 \mathrm{~Hz}), 6.37(1 \mathrm{H}, \mathrm{t}, J=15.8 \mathrm{~Hz}), 7.30-7.58(5 \mathrm{H}, \mathrm{m}), 7.58-7.90$ (4H, m), 7.59$7.61(2 \mathrm{H}, \mathrm{m}), 7.90(1 \mathrm{H}, \mathrm{d}, J=16 \mathrm{~Hz}), 8.04(2 \mathrm{H}, \mathrm{d}, J=8.7 \mathrm{~Hz}) .{ }^{13} \mathrm{C}$ NMR $\left(100 \mathrm{MHz}, \mathrm{CDCl}_{3}\right)$ : 37.2 , 67.7, 105.6, 117.6, 123.4, 128.4, 129.1, 130.4, 130.6, 132.2, 134.3, 145.7, 166.9, 168.3.

2-Phthalimidoethyl 3,4,5-trimethoxycinnamate (4n). ${ }^{32} \mathrm{H} \mathrm{NMR}\left(400 \mathrm{MHz}, \mathrm{CDCl}_{3}\right): 1.24(3 \mathrm{H}$, $\mathrm{t}, J=7.3 \mathrm{~Hz}), 3.01(2 \mathrm{H}, \mathrm{qt}, J=7.3 \mathrm{~Hz}), 6.64(1 \mathrm{H}, \mathrm{d}, J=16 \mathrm{~Hz}), 7.26-7.29(2 \mathrm{H}, \mathrm{m}), 7.45-7.43$ $(3 \mathrm{H}, \mathrm{m}), 7.59-7.61(2 \mathrm{H}, \mathrm{m}), 7.90(1 \mathrm{H}, \mathrm{d}, J=16 \mathrm{~Hz}), 8.04(2 \mathrm{H}, \mathrm{d}, J=8.7 \mathrm{~Hz}) ;{ }^{13} \mathrm{C} \mathrm{NMR}(100$ $\mathrm{MHz}_{\mathrm{CDCl}}$ ): 37.4, 56.4, 67.6, 105.6, 116.9, 123.4, 130.0, 132.2, 132.3, 134.0, 145.7, 153.6, 167.0, 168.4 . 
1-Naphthyl cinnamate (4o). ${ }^{\text {lit. } 1}{ }^{1} \mathrm{H}$ NMR (400 MHz, $\left.\mathrm{CDCl}_{3}\right): 1.24(3 \mathrm{H}, \mathrm{t}, J=7.3 \mathrm{~Hz}), 3.01(2 \mathrm{H}$, qt, $J=7.3 \mathrm{~Hz}), 6.64(1 \mathrm{H}, \mathrm{d}, J=16 \mathrm{~Hz}), 7.26-7.29(2 \mathrm{H}, \mathrm{m}), 7.45-7.43(3 \mathrm{H}, \mathrm{m}), 7.59-7.61(2 \mathrm{H}$, m), $7.90(1 \mathrm{H}, \mathrm{d}, J=16 \mathrm{~Hz}), 8.04(2 \mathrm{H}, \mathrm{d}, J=8.7 \mathrm{~Hz}) ;{ }^{13} \mathrm{C} \mathrm{NMR}\left(100 \mathrm{MHz}, \mathrm{CDCl}_{3}\right): 117.0$, $118.1,121.3,125.4,126.0,126.4,127.0,128.0,128.4$, 129.0, 130.8, 134.2, 134.7, 146.7, 147.0, 165.4 .

Phenyl $\boldsymbol{\alpha}$-phenylcinnamate (4p). ${ }^{\text {lit.33 }}{ }^{1} \mathrm{H} \mathrm{NMR}\left(400 \mathrm{MHz}, \mathrm{CDCl}_{3}\right): 1.24(3 \mathrm{H}, \mathrm{t}, J=7.3 \mathrm{~Hz}), 3.01$ $(2 \mathrm{H}, \mathrm{qt}, J=7.3 \mathrm{~Hz}), 6.64(1 \mathrm{H}, \mathrm{d}, J=16 \mathrm{~Hz}), 7.26-7.29(2 \mathrm{H}, \mathrm{m}), 7.45-7.43(3 \mathrm{H}, \mathrm{m}), 7.59-7.61$ $(2 \mathrm{H}, \mathrm{m}), 7.90(1 \mathrm{H}, \mathrm{d}, J=16 \mathrm{~Hz}), 8.04(2 \mathrm{H}, \mathrm{d}, J=8.7 \mathrm{~Hz}) ;{ }^{13} \mathrm{C} \mathrm{NMR}\left(100 \mathrm{MHz}, \mathrm{CDCl}_{3}\right): 121.6$, $125.7,128.0,128.3,128.7,129.3,129.4,129.8,129.9,130.8,132.0,134.5,135.5,142.0,151.2$, 162.0 .

\section{Conclusions}

The described procedure provides a clean, fast and useful alternative to prepare aryl and 2phthalimidoethyl cinnamates. In general, the use of supported WD acid affords very good yield in lower reaction times and furthermore, allows for their easy separation and recovering. The filtered catalyst is ready for immediate reutilization, with no decrease of its activity being noted. Advantages of this methodology are: operational simplicity, use of a non-corrosive and reusable catalyst, mild conditions, short reaction times and very good yields. The use of the solid catalyst allows one to avoid the use of acid chloride or $\mathrm{SOCl}_{2}$, pyridine or coupling agents, contributing to a reduction of eventually dangerous waste.

\section{Acknowledgements}

We thank Fundación Antorchas, Universidad Nacional de La Plata, and Agencia Nacional de Promoción Científica y Tecnológica (Argentina) for financial support. H.J.T, G.P.R. and G.B. are members of CONICET (Argentina); D. M. R. also thanks CONICET for a grant.

\section{References and Notes}

1. Gobec, S.; Sova, M.; Kristan, K.; Rizner, T. L. Bioorg. Med. Chem. Lett. 2004, 14, 3933.

2. Shankaran, K.; Sloan, C. P.; Snieckus, V. Tetrahedron Lett. 1985, 26, 6001.

3. Pinto, C. G. A.; Silva, A. M. S.; Cavaleiro, J. A. S.; Foces-Foces, C.; Llamas-Saiz, A. L.; Jegerovic, N.; Elguero, J. Tetrahedron 1999, 55, 10187.

4. Zhu, J.; Majikina, M.; Tawata, S. Biosci. Biotechnol. Biochem, 2001, 65, 161.

5. Womack, E. B.; McWhirter, J. Org. Synth. coll. Vol. III, 1955, 714.

6. Isaacs, N. S.; Najem, T. S. J. Chem. Soc. Perkin Trans. 2 1988, 557. 
7. Mali, R.; Papalkar, A.S. J. Chem. Res. (S) 2003, 603 and references cited therein.

8. Aldrich Catalog 2005-2006, Sigma-Aldrich Co.: Milwaukee, 2005; p. 280.

9. Nakayama, M.; Sato, A.; Ishihara, K.; Yamamoto, H. Adv. Synth. Catal. 2004, 346, 1275.

10. Sinha, A.; Sharma, A.; Swaroop, A.; Kumar, V. Tetrahedron 2007, 63, 1000.

11. Arabi, M.; Mohammadpour Amini, M.; Abedini, M.; Nemati, A.; Alizadeh, M. J. Mol. Catal. A, Chemical 2003, 200, 105.

12. Alizadeh, M. H.; Kermany, T.; Tayebee, R. Monatsh. Chem. 2007, 138, 165.

13. Kozhevnikov, I.V. Chem. Rev. 1998, 98, 171.

14. Tungler, A.; Sípos, E.; Háda, V. ARKIVOC 2004, (vii), 223.

15. Baronetti, G.; Briand, L.; Sedran, U.; Thomas, H. Appl. Catal. A 1998, 172, 265.

16. Sambeth, J.; Baronetti, G.; Thomas, H. J. Mol. Catal. A, Chemical 2003, 191, 35.

17. Tarlani, A.; Riahi, A.; Abedini, M.; Amini, M. M.; Muzart, J. Catal. Commun. 2007, 8, 1153.

18. Li, G.; Gu, Y.; Ding, Y.; Zhang, H.; Wang, J.; Gao, Q.; Yan, L.; Suo, J. J. Mol. Catal. A: Chemical 2004, $218,147$.

19. Romanelli, G. P.; Bennardi, D.; Ruiz, D. M.; Baronetti, G.; Thomas, H. J.; Autino, J. C. Tetrahedron Lett. 2004, 45, 8935 and references cited.

20. Timofeeva, M. N. Appl. Catal. A: General 2003, 256, 19.

21. Briand, L. E.; Baronetti, G. T.; Thomas, H. J. Appl. Catal. A: General 2003, 256, 37.

22. Ben-Daniel, R.; Khenkin, A. M.; Neumann, R. Chem. Europ. J. 2000, 6, 3722.

23. Li, G.; Wang, B.; Wang, J.; Ding, Y.; Yan, L.; Suoa, J. J. Mol. Catal. A 2005, 236, 72.

24. Romanelli, G. P.; Autino, J. C.; Baronetti, G.; Thomas, H. J. Synth. Commun. 2004, 34, 3909.

25. Romanelli, G. P.; Ruiz, D. M.; Bideberripe, H. P.; Autino, J. C.; Baronetti, G.; Thomas, H. J. Arkivoc 2007, (i), 1.

26. Kaitner, B.; Stilinovic, V., Acta Cryst. 2007, E63, o4347.

27. Pattanaargson, S.; Hongchinnagorn, N.; Hirunsupachot, P.; Sritana-anant, Y.; Photochemistry and Photobiology 2004, 80, 322.

28. Ishidoya, M.; Nakamichi, T., European Patent Aplication, 1989, EP0320954.

29. Nishikubo, T.; Iizawa, K.; Takahashi, E.; Nono, F.; Patent Abstracts of Japan, 1984, JP59216138.

30. Kang, H.; Rho, H.; Hwang, J.; Oh, S.; Chem. Pharm. Bull. 2003, 51, 1085.

31. Harris, S.; Christiansen, W.; J. Am. Pharm. Assoc. 1935, 24, 553.

32. Kristan, K; Starčevic, S.; Brunskole, M.; Rižner, T.L.; Gobec, S.; Molecular and Cellular Endocrinology 2006, 248, 239.

33. Nicolaus, R.; Ann. Chim. Applicata 1949, 39, 542. 